\title{
AAV-mediated gene transfer of human pigment epithelium-derived factor inhibits Lewis lung carcinoma growth in mice
}

\author{
SHA-SHA HE, HUA-SHAN SHI, TAO YIN, YONG-XIA LI, SHUN-TAO LUO, \\ QIN-JIE WU, LIAN LU, YU-QUAN WEI and LI YANG \\ State Key Laboratory of Biotherapy, West China Hospital, Sichuan University, Chengdu 610041, P.R. China
}

Received October 14, 2011; Accepted December 2, 2011

DOI: $10.3892 / o r .2012 .1621$

\begin{abstract}
Pigment epithelium-derived factor (PEDF) is the most potent inhibitor of angiogenesis in the mammalian eye, and mechanisms through which PEDF exerts its antitumour activity have recently been defined. The aim of our research was to evaluate the ability of adeno-associated virus (AAV) vector-mediated transfer of human PEDF to inhibit Lewis lung carcinoma (LCC) cell growth. Intratumoural injection of AAV-PEDF caused significant reduction of the tumour volume and prolonged the survival time of mice bearing LLC cells, which were associated with decreased microvessel density and increased apoptosis in the tumours. AAV vectors represent a very promising tool for cancer gene therapy. No noticeable toxicity concerning AAV was detected as inferred from monitoring changes in animal body weight as well as basic organ structure and histological morphology, and by analyzing mouse liver and kidney function. Our findings indicate that AAV-mediated PEDF gene expression may offer an active approach to inhibit LLC growth and that treatment with AAV-PEDF may provide a promising therapeutic strategy in lung cancer treatment.
\end{abstract}

\section{Introduction}

Extensive research has established that neoangiogenesis plays a pivotal role in solid malignant tumour growth and metastasis (1-3). Tumours cannot exceed a few millimetres in diameter

Correspondence to: Dr Professor Li Yang, State Key Laboratory of Biotherapy, West China Hospital, Sichuan University, Keyuan Road 4, Chengdu, Sichuan 610041, P.R. China

E-mail: yl.tracy73@gmail.com

Abbreviations: PEDF, pigment epithelium-derived factor; AAV, adeno-associated virus; MVD, microvessel density; LLC, Lewis lung carcinoma; VEGF, vascular endothelial growth factor; HUVECs, human umbilical vein endothelial cells; NS, normal saline; VEGF-R1, vascular endothelial growth factor-receptor 1; VEGF-R2, vascular endothelial growth factor-receptor 2

Key words: AAV-PEDF, LLC, tumour, apoptosis, angiogenesis without the development of a neovasculature supply. Thus, antiangiogenesis therapy is a potentially promising tumouristatic approach (4-6). However, evidence has emerged that angiogenesis is tightly regulated by a balance of activating and inhibiting factors $(7,8)$. Therefore, long-term overexpression of angiogenesis suppressors may be required for effectively controlling tumour proliferation through counteracting tumour-induced angiogenesis.

Pigment epithelium-derived factor (PEDF) is a $50-\mathrm{kDa}$ secreted glycoprotein that belongs to the serine protease inhibitor superfamily but lacks protease inhibitor activity (9-11). PEDF was first identified and purified from the conditioned medium of cultured human neonatal retinal pigment epithelial cells $(9,12)$. This factor is involved in multiple and varied biological activities (13), which makes it an appealing potential treatment for Lewis lung carcinoma (LCC). With regard to its antiangiogenic activity, PEDF is more potent than any other endogenous inhibitors of neovascularisation $(14,15)$; this property makes PEDF an excellent candidate for LLC treatment as a form of targeted gene therapy. The antiangiogenic potency of PEDF has been shown to inhibit tumour angiogenesis in several preclinical cancer models (16-27). In addition, PEDF is thought to exert its antiangiogenic activity through two major pathways, namely, endothelial cell apoptosis via activation of the Fas/ Fas-L death pathway (28), and disruption of the crucial balance between pro- and anti-angiogenic factors, via downregulation of vascular endothelial growth factor (VEGF) expression (29-31). Furthermore, a few recent reports indicate that PEDF not only acts to halt angiogenesis, but also has the ability to increase apoptosis in tumours $(16-18,26)$. This apoptotic activity is likely due to a distinct functional epitope on the PEDF protein (20).

Successful antiangiogenic therapy requires efficient and continuous secretion of the candidate protein for long periods of time. Gene transfer is usually utilized as an effective strategy for chronic delivery of antiangiogenic factors. Adenoassociated virus (AAV) vectors represent a very promising tool for cancer gene therapy because they are capable of sustained, long-term gene expression, non-pathogenicity, low immunogenicity, and they lack cytotoxicity (32-34).

In this study, we constructed AAV vectors that express PEDF in order to investigate the effect of AAV-mediated intratumoural PEDF expression on LLC tumour suppression. 


\section{Materials and methods}

Cell lines and culture. HUVECs were isolated and cultured in DMEM medium (Gibco-BRL, NY, USA) supplemented with $20 \%$ FBS and $100 \mathrm{~g} / \mathrm{ml}$ bovine fibroblast growth factor (BFGF). LLC cells were obtained from the American Type Culture Collection (ATCC, Rockville, MD, USA) and cultured in DMEM medium (Gibco-BRL) supplemented with $10 \%$ FBS and $100 \mu \mathrm{g} / \mathrm{ml}$ amikacin.

$A A V$-PEDF preparation. AAV-PEDF was constructed using $\mathrm{CMV}$ as the promoter. cDNAs containing full-length human PEDF sequences under the CMV promoter were cloned. The construct sequence was confirmed via DNA sequencing (Invitrogen Inc., Shanghai). A control virus containing a green fluorescent protein cDNA under the same promoter (AAV-EGFP) was also detected by DNA sequencing (Invitrogen Inc.). Packaging and purification of rAAV particles were performed as previously described (35).

Cell infection with AAV-PEDF. LLC cells were seeded in 6 -well plates. After an overnight culture, the cells were infected with AAV-PEDF and AAV-EGFP viruses at a multiplicity of infection (MOI) of $1 \times 10^{5}$ infectious particles/cell. The cells and supernatants were collected after $72 \mathrm{~h}$.

Western blot analysis. LLC cells seeded in the 6-well plates were harvested and resuspended in lysis buffer while the LLC tumours were grinded and then lysed with RIPA solution, respectively. Equal amounts of protein were separated by SDS-polyacrylamide gel electrophoresis (PAGE) and then electrotransferred onto a polyvinylidene difluoride membrane (PVDF). Blots were probed with a goat anti-human PEDF monoclonal antibody (1:1000, mAb; R\&D Systems, Boston, MA, USA) plus a secondary biotinylated antibody against goat IgG (1:10,000, ZSGB-BIO, Beijing, China). Immunoreactivity was detected using an enhanced chemiluminescence (ECL) detection system (Pierce, Rockford, IL, USA).

Animal experiments. Male C57BL/6 mice were purchased from the Experimental Animal Centre at Sichuan University. All animal experimental procedures were approved by the West China Hospital Cancer Centre's Animal Care and Use Committee. Aliquots of LLC cells $\left(5 \times 10^{5}\right)$ were subcutaneously inoculated into the mice. When the average tumour volume reached $90-100 \mathrm{~mm}^{3}$ in size, the mice were randomly divided into 3 groups. Each mouse in the AAV-PEDF group was treated with an intratumoural injection of $2 \times 10^{10}$ AAV-PEDF virus particles. The mice in the control groups received $2 \times 10^{10}$ AAV-EGFP particles or normal saline (NS). Tumour sizes and animal weights were measured every three days. The tumour volumes $\left(\mathrm{mm}^{3}\right)$ were calculated according to the following formula: (length $\mathrm{x}$ width ${ }^{2} \mathrm{x}$ 0.52). Mouse sera were collected for liver and kidney function analyses on the fifteenth day post-treatment using an AU7020 Automatic Biochemical Analyzer (Hitachi).

TUNEL assay, immunohistochemistry analysis and $H \& E$ staining. Apoptotic tumour cells were determined by the DeadEnd colorimetric terminal deoxynucleotidyl transferase-mediated dUTP nick-end labelling (TUNEL) System (Promega Corp., Madison, USA) and the caspase-3 immunohistochemical assay. Prepared tumour cryosections were incubated with primary anti-human PEDF antibody (1:200, mAb; R\&D Systems) overnight, then with biotinylated anti-goat IgG secondary antibody (ZSJQ Biotechnology) and finally with diaminobenzidine (DAB; ZSJQ Biotechnology) as a substrate for visualization of the antigen-antibody complex. Frozen tumour specimens were analyzed by CD31 immunohistochemistry. The microvessel density was quantified using the reported method of Weidner et al (36). Paraffin sections were also stained with haematoxylin and eosin (H\&E) to observe the structure and histological morphology of the tumours and basic organs.

Alginate-encapsulated tumour cell and tube formation assay. LLC cells were resuspended in alginate solution. LLC cells in alginate solution were dropped into a swirling $0.25 \mathrm{M}$ $\mathrm{CaCl}_{2}$ solution to prepare alginate beads $\left(1 \times 10^{5}\right.$ cells/bead $)$. Male C57Bl/6 mice were implanted s.c. with alginate beads into the back ( 1 bead/side). The next day, the mice were treated with i.v. administration of $2 \times 10^{10}$ particles AAV-PEDF per mouse, or $2 \times 10^{10}$ particles AAV-EGFP per mouse or with NS.

On Day 11 after treatment, $0.1 \mathrm{ml}$ of $1 \%$ FITC-dextran solution $(100 \mathrm{mg} / \mathrm{kg})$ was injected i.v. into the tail vein of the mice. Alginate beads were photographed and rapidly removed 20 min after FITC-dextran injection. The beads were then vortexed and centrifuged in tubes containing $2 \mathrm{ml} \mathrm{NS}$ and the supernatant fluorescence was measured.

The tube formation assay was performed using Matrigel (BD Biosciences, San Jose, CA) that was thawed overnight at $4^{\circ} \mathrm{C}$. Pre-chilled 24-well plates were coated with $300 \mu \mathrm{l} /$ well of Matrigel (BD Biosciences). HUVECs were seeded in each well at a concentration of $1 \times 10^{5}$ cells, and then treated with conditioned medium (CM) from LLC cells infected in vitro with AAV-PEDF, AAV-EGFP or NS. Tubule branches were photographed $6 \mathrm{~h}$ after incubation.

Statistical analysis. Values are presented as means \pm SD Statistical analysis was conducted using the SPSS program (version 18.0, SPSS Inc., Chicago, IL, USA). The statistical significances were calculated by one-way ANOVA. The Kaplan-Meier method was used to evaluate survival curves and survival rate among groups. A P-value $<0.05$ was considered significant.

\section{Results}

PEDF gene expression in vivo and in vitro. To confirm PEDF gene expression in vivo and in vitro, western blotting was performed. PEDF protein was detected only in LLC tumours from AAV-PEDF treated mice (Fig. 2B). Human PEDF was detected as a single $50 \mathrm{kDa}$ band in AAV-PEDF transduced cells (Fig. 2C). No PEDF was found in the lysates from AAV-EGFP-transduced or NS-treated cells. Seven days after inoculation, tumours were injected with AAV-EGFP, AAV-PEDF, or NS, and only the cytoplasm of LLC cells treated with AAV-PEDF clearly was stained for intratumoural PEDF (Fig. 2A). These results demonstrate successful PEDF gene expression in vivo and in vitro. 

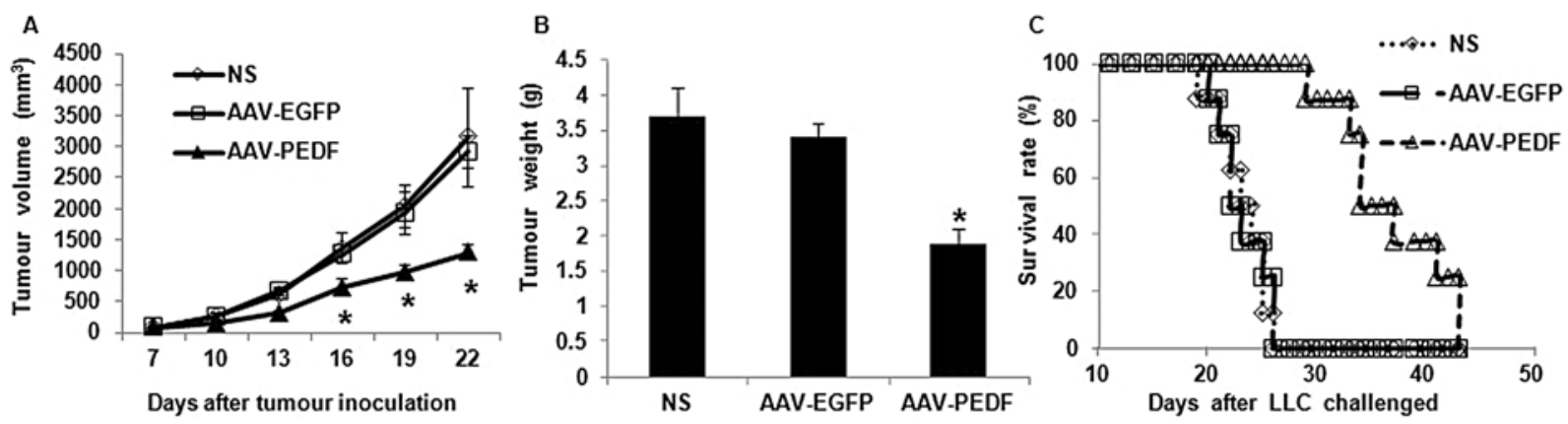

Figure 1. Antitumour efficacy of AAV-PEDF in vivo. (A) LLC cells were inoculated into C57BL/6 mice. When the average tumour size reached $90-100 \mathrm{~mm}^{3}$, the mice received an intratumoural injection of AAV-PEDF, AAV-EGFP or normal saline (NS). Tumour volumes were measured and calculated. (B) Mice were sacrificed on Day 15 after LLC inoculation and tumours were weighed. (C) Survival curves of mice. Significant increases in survival rates and prolonged survival times were observed in AAV-PEDF-treated mice. $\mathrm{N}=8$ per group. ${ }^{*} \mathrm{P}<0.05$ compared to AAV-EGFP or the NS control groups.

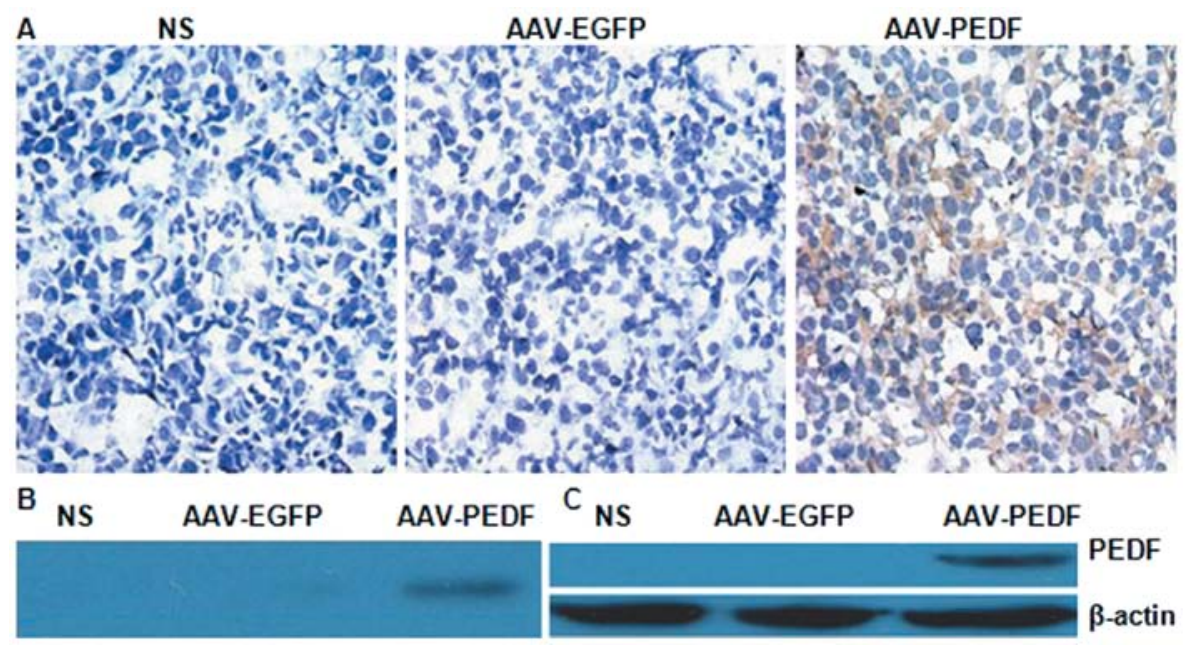

Figure 2. PEDF gene expression in vivo and in vitro. Mice were euthanized on day 15 post-treatment and (A) tumours were processed for immunohistochemistry analysis (original magnification, $\mathrm{x} 400$ ). Only the cytoplasm of LLC cells treated with AAV-PEDF was stained for intratumoural PEDF. (B) PEDF protein $(50 \mathrm{kDa})$ was detected in AAV-PEDF, but not AAV-EGFP or normal-saline (NS)-treated LLC tumours. (C) Human PEDF was detected as a single band of $50 \mathrm{kDa}$ in AAV-PEDF-infected cells, but not in AAV-EGFP-infected or NS-treated cells.

Intratumoural treatment of AAV-PEDF suppresses tumour growth and prolongs mouse survival rate. Established subcutaneous LLC tumours in the C57BL/6 mouse model were used to investigate the antitumoural efficacy of AAV-PEDF by intratumoural injection. There were no significant differences in size between those tumours receiving NS vs. AAV-EGFP (Fig. 1A). In contrast, the growth of AAV-PEDF-treated LLC tumours was significantly inhibited compared with either AAV-EGFP or NS (Fig. 1A, P<0.05). The maximum tumour growth inhibition was observed on day 15 after treatment (56 and 58\% inhibition, respectively, compared with mice treated with AAV-EGFP or NS, Fig. 1A). Tumour weights in the AAV-PEDF group were significantly lower than either the AAV-EGFP or NS groups (Fig. 1B, $\mathrm{P}<0.05$ ). In addition, intratumoural treatment of AAV-PEDF resulted in prolonged animal survival (Fig. 1C, $\mathrm{P}<0.05$ ).

AAV-PEDF treatment decreases microvessel density and increases apoptosis in tumour tissue. Tumours from AAV-PEDF-treated animals showed marked reduction in microvessel density (MVD) (Fig. 3A). Quantitative analysis showed a 71 and $73 \%$ reduction in intratumoural MVD in AAV-PEDF treated animals compared with control animals receiving AAV-EGFP and NS, respectively (Fig. 3B). There was no significant difference in vessel density between tumours that received NS or AAV-EGFP (Fig. 3B; NS vs. AAV-EGFP, $P>0.1$.

More apoptotic cells in tumour tissue were observed in AAV-PEDF-treated animals than in AAV-EGFP- or NS-treated animals (Fig. 4A). The average percentage of apoptosis in the AAV-PEDF group was significantly increased compared with that in the AAV-EGFP and NS groups (Fig. 4B; $\mathrm{P}<0.05$ ). Furthermore, there were more cell nuclei stained in the AAV-PEDF group compared with the other groups (Fig. 4C and $\mathrm{D} ; \mathrm{P}<0.05)$. As shown in Fig. 4E, histological analysis revealed that AAV-PEDF-treated tumours had increased cell death necrosis.

$A A V-P E D F$ inhibition of angiogenesis in vitro and in vivo. Endothelial cells form tube and capillary-like structures on a Matrigel membrane through a process involving attachment, alignment, and migration. Treatment with conditioned medium 

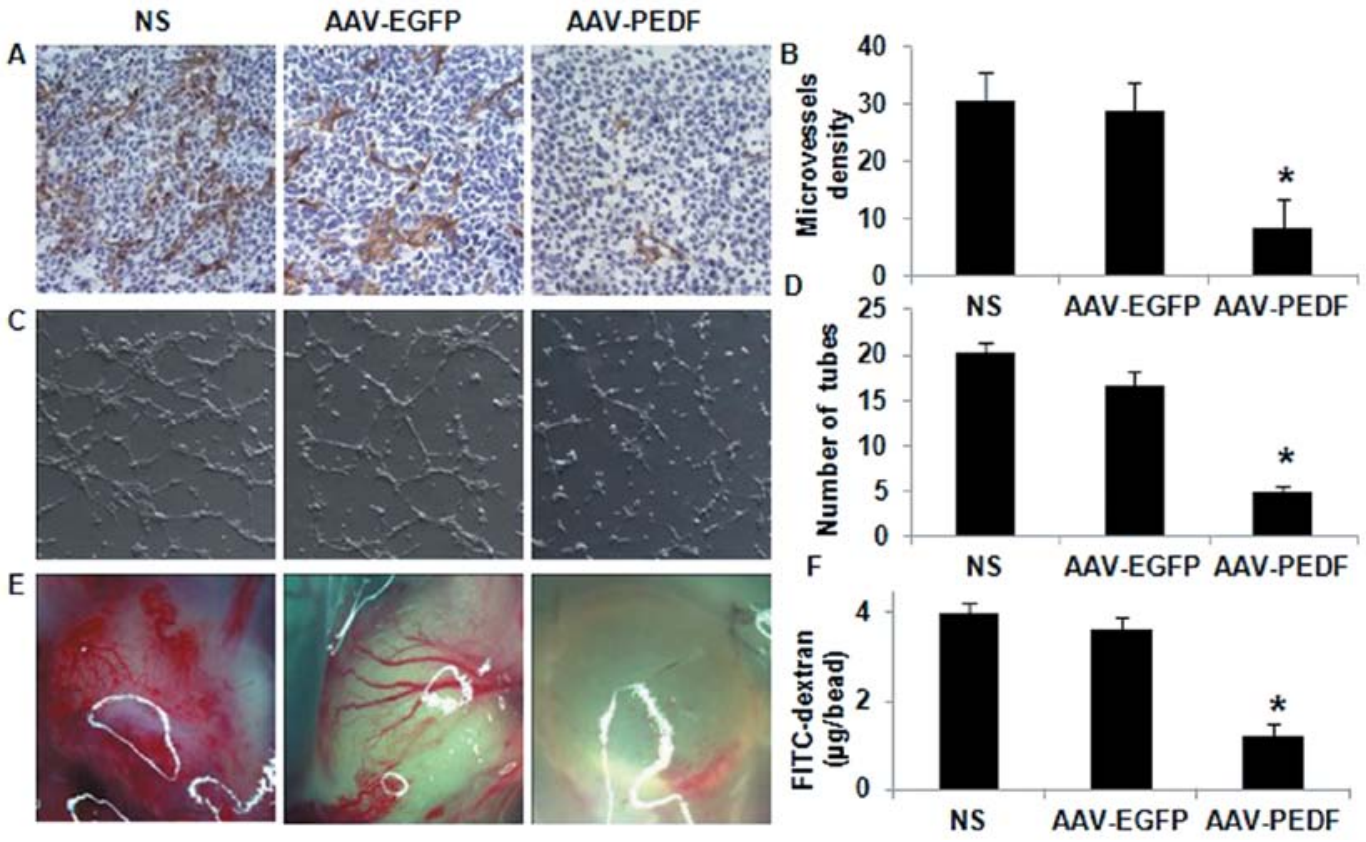

Figure 3. CD31 immunohistochemistry, tube formation, and Alginate-encapsulated tumour cell assay. (A) Micrographs show tumour tissue sections stained with anti-CD31 antibody (original magnification, x400) after NS, AAV-EGFP, or AAV-PEDF treatment. (B) Quantitative results of microvessel density (MVD) in tumour tissue. The AAV-PEDF group showed a significant decrease in MVD compared to control groups ("P $<0.05$ ). Endothelial cells form tube and capillary-like structures on a Matrigel membrane through a process involving attachment, alignment and migration. (C) Treatment with conditioned medium (CM) from LLC cells infected in vitro with AAV-PEDF significantly decreased this process (original magnification, $x 100)$. (D) The tube formations were quantified by counting the number of connecting branches between 2 discrete endothelial cells ( $\mathrm{P}<0.05)$. (E) Photographs show alginate bead surfaces from different groups. (F) FITC-dextran uptake in the tumour was significantly decreased in mice treated with AAV-PEDF compared to the control groups $($ ( $\mathrm{P}<0.05)$.
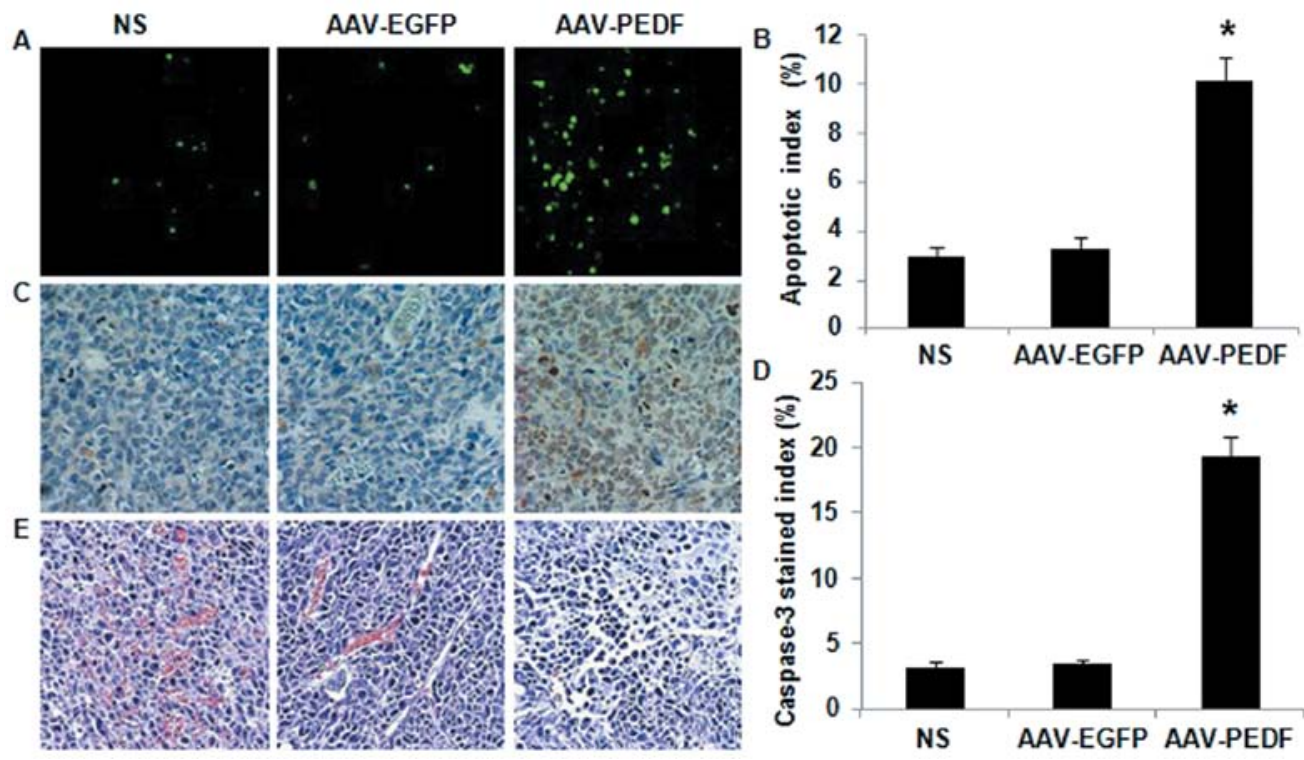

Figure 4. TUNEL, caspase-3 immunohistochemical assay, and histological staining for tumour tissue. (A) Apoptotic cells within LLC tumours were detected by the TUNEL assay (original magnification, $\mathrm{x} 400$ ). (B) The apoptotic index (\%) of LLC tumours treated with NS, AAV-EGFP, and AAV-PEDF ("P<0.05). (C) Micrographs show tumour tissue sections stained with anti-caspase-3 antibody (original magnification, $x 400$ ) after NS, AAV-EGFP or AAV-PEDF treatment. (D) The caspase-3 stained index (\%) of tumours treated with NS, AAV-EGFP, and AAV-PEDF ("P<0.05). (E) LLC tumours from NS, AAV-EGFP, and AAV-PEDF treated mice were stained with H\&E (original magnification, $\mathrm{x} 400$ ); noticeable necrosis was observed in tumours from AAV-PEDF treated mice.

(CM) from LLC cells infected in vitro with AAV-PEDF significantly decreased this process (Fig. 3C). Quantitative analysis showed that AAV-PEDF restricted tube formation by 71 and $76 \%$, respectively, in comparison to the AAV-EGFP or NS controls (Fig. 3D; P<0.01).
Alginate implants in AAV-PEDF-treated animals exhibited less vascularisation than AAV-EGFP- or NS-treated animals (Fig. 3E). We observed that the accumulation of FITC-dextran at the implant site in the AAV-PEDF group was significantly lower compared to the other groups (Fig. 3F, $\mathrm{P}<0.05$ ). 

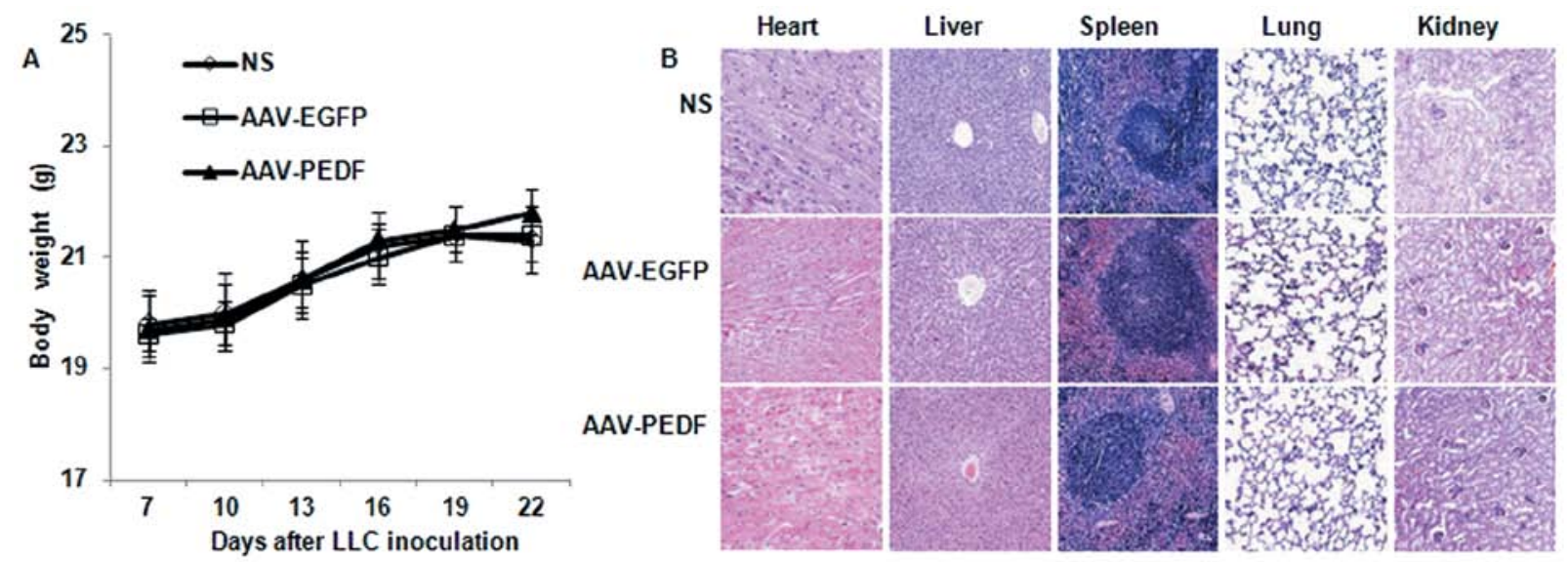

Figure 5. Changes in body weight and histological staining for basic organs. (A) There was no difference in body weight among the treatment groups. (P>0.1) (B) Basic organs from NS-, AAV-EGFP-, and AAV-PEDF-treated mice were stained with H\&E (original magnification, x200). There was no noticeable pathological change in tumours from AAV-EGFP- and AAV-PEDF-treated mice compared with NS control.
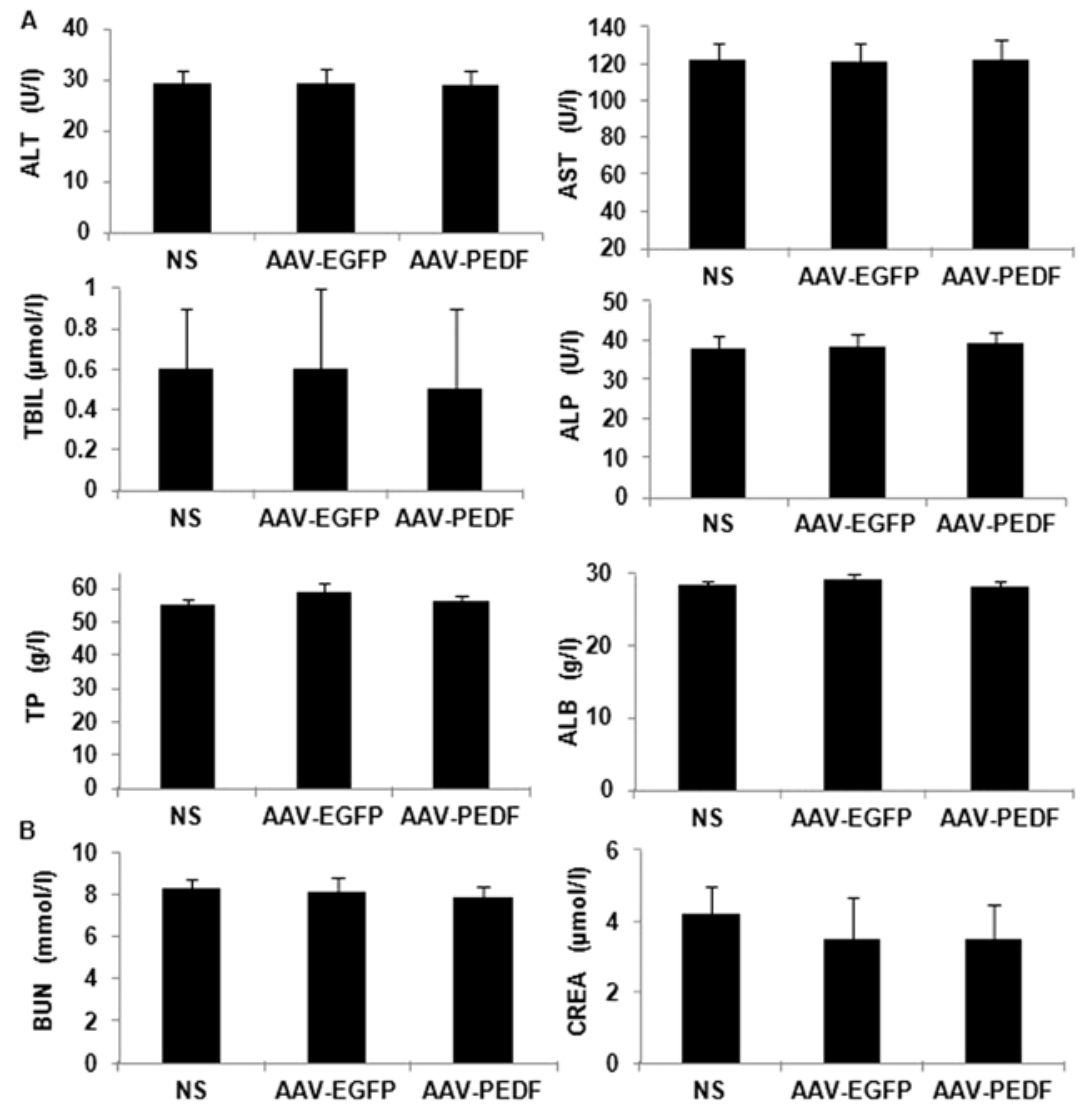

Figure 6. Mouse liver and kidney function. (A) There was no difference in mouse liver function of ALT, AST, TBIL, ALP, TP, and ALB in the respective treatment groups $(\mathrm{P}>0.1)$. (B) There was also no difference in the kidney function as measured by BUN and CREA $(\mathrm{P}>0.1)$.

The data confirmed that AAV-PEDF-mediated PEDF gene transfer and expression could suppress tumour angiogenesis in the studied tumour model.

$A A V$ toxicity in mice. We evaluated AAV toxicity in mice by monitoring changes in mouse weight, basic organ structure, and histological morphology, and analyzing mouse liver and kidney function. No significant difference was observed in body weight among AAV-EGFP-, AAV-PEDF- or NS-treated mice (Fig. 5A, P>0.1). Liver and kidney function data are shown in Fig. 6 ( $\mathrm{P}>0.1)$. In addition, no apparent pathological changes were observed in the heart, liver, spleen, lung, and kidney tissue from the different groups as indicated by $\mathrm{H} \& \mathrm{E}$ staining (Fig. 5B).

\section{Discussion}

Angiogenesis, the complex biological process by which new blood vessels develop from pre-existing ones, is known to play an essential role in supporting progressive tumour 
growth (1-3). Therefore, targeting neoangiogenesis or signals that promote neovessel growth is a promising anticancer therapeutic strategy (4-6). However, neovasculature growth is controlled by maintaining a balance between pro- and antiangiogenic factors $(7,8)$. For this reason, overexpression of anti-angiogenesis factors could be excellent therapeutic tools in combating tumour angiogenesis.

Combating lung cancer remains a major clinical challenge. Existing therapeutic protocols are very disappointing. Previous studies have demonstrated the efficacy of anti-angiogenesis therapy (4-6). In this study, we demonstrated that overexpression of PEDF mediated by the AAV vector exerts a remarkable suppression of tumour growth and prolongs animal survival in a C57BL/6 mouse model. The tumours treated with a single intratumoural injection of AAV-PEDF began to grow more slowly than the other two groups on Day 6 after treatment with maximum tumour growth inhibition observed on Day 15 after treatment (56\% and 58\% inhibition, respectively, compared with AAV-EGFP- or NS-treated mice). Tumour growth suppression was related to decreased microvessel density and increased apoptosis in AAV-PEDF-treated tumours. A 73\% decreased MVD in the AAV-PEDF-treated tumours (Fig. 3A and B) closely paralleled the $58 \%$ reduction in tumour size, implying a direct relationship between lung carcinoma vascularity and growth. The antiangiogenic activity of PEDF was further demonstrated by tube formation and the alginate-encapsulated tumour cell assay (Fig. 3C-F). The average percentage of apoptosis in the AAV-PEDF group (10\%) was significantly increased, compared with the AAV-EGFP (3\%) and NS (3\%) groups (Fig. 4B; $\mathrm{P}<0.05)$. These observations suggest that hindering the vascular supply to a tumour significantly curbs its ability to grow and that restriction of tumour angiogenesis caused an increase in tumour cell apoptosis in AAV-PEDF treated mice.

The mechanisms by which PEDF reduces neovascularisation are still largely unknown. Nevertheless, 2 major pathways have been implicated, namely, endothelial cell apoptosis via activation of the Fas/Fas-L death pathway (28) and disruption of the crucial balance between pro- and anti-angiogenic factors, in particular downregulation of VEGF expression (29-31). Whereas pro-angiogenic factors stimulate endothelial cells that express Fas, PEDF increases FasL expression on the endothelial cell surface. A caspase-dependent apoptotic cascade is subsequently initiated, resulting in endothelial cell death. Another mechanism underlying the antiangiogenic property of PEDF is that it also has an inhibitory effect on VEGF-induced angiogenesis. A study by Cai et al reported that PEDF activates $\gamma$-secretase-dependent cleavage of the VEGF-R1 C terminus, which consequently modulates angiogenic signalling via VEGF-R2 (31). Exogenous PEDF downregulated VEGF expression at both the mRNA and protein levels in the MG63 osteosarcoma cell line (26).

In this present study, our data indicated that PEDF not only acts to halt angiogenesis, but also has the ability to increase apoptosis in tumours. However, the mechanisms of PEDFmediated apoptosis in tumours are still poorly understood. The apoptotic activity is likely due to a distinct functional epitope on the PEDF protein that was discovered by Filleur et al, who reported increases in prostate cancer cell death in vitro with the 34-mer peptide, but not with the 44-mer (20). Significant tumour cell apoptosis with full-length PEDF treatment in vitro has been observes in melanoma and osteosarcoma cells $(16,26)$. This effect may be mediated by the Fas/Fas-L cascade similar to endothelial cell apoptosis, as it was reversed by neutralizing antibodies against Fas-L $(16,26)$.

AAV has several excellent advantages making it a particularly promising vector for gene therapy. AAV has long-term transgene expression in experimental gene therapy methods compared with other viral vectors (37). More importantly, AAV is a non-pathogenic virus and has low immunogenicity because the genes encoding the wild-type protein are absent, and it has a replication-defective nature (32-34). In our study, we did not detect AAV-induced toxicity as measured by calculating the change in animal body weight, observing basic organ structure and histological morphology, and analyzing mouse liver and kidney function (Figs. 5 and 6). Several recent reports have demonstrated the therapeutic efficiency of PEDF viral vector intratumoural injections $(20,38,39)$. Intratumoural gene therapy allows for selective expression within tumour cells contributing to higher intratumoural concentrations while maintaining normal systemic levels, thus limiting the number of adverse reactions. AAV vectors in gene therapy could be relatively safe and efficient.

In conclusion, our results indicate for the first time that AAV-PEDF by intratumoural injection can provide an effective protocol for LLC treatment in a C57BL/6 mouse model. Mechanisms of PEDF-mediated reduction of LLC tumour growth include both inhibition of tumour angiogenesis and stimulation of tumour cell apoptosis.

\section{Acknowledgements}

This study was funded by the National Key Basic Research Program (973 Program) of China (2010CB529900).

\section{References}

1. Hanahan D and Weinberg RA: The hallmarks of cancer. Cell 100: $57-70,2000$

2. Folkman $\mathbf{J}$ and Ingber $D$ : Inhibition of angiogenesis. Semin Cancer Biol 3: 89-96, 1992

3. Bicknell R and Harris AL: Anticancer strategies involving the vasculature: vascular targeting and the inhibition of angiogenesis. Semin Cancer Biol 3: 399-407, 1992.

4. Augustin HG: Translating angiogenesis research into the clinic: the challenges ahead. Br J Radiol 76: 3-10, 2003.

5. Shimizu K and Oku N: Cancer anti-angiogenic therapy. Biol Pharm Bull 27: 599-605, 2004.

6. Purow B and Fine HA: Progress report on the potential of angiogenesis inhibitors for neuro-oncology. Cancer Invest 22: 577-587, 2004.

7. Conway EM, Collen D and Carmeliet P: Molecular mechanisms of blood vessel growth. Cardiovasc Res 49: 507-521, 2001.

8. Yancopoulos GD, Davis S, Gale NW, Rudge JS, Wiegand SJ and Holash J: Vascular-specific growth factors and blood vessel formation. Nature 407: 242-248, 2000.

9. Tombran-Tink J, Chader GG and Johnson LV: PEDF: A pigment epithelium derived factor with potent neuronal differentiative activity. Exp Eye Res 53: 11-14, 1991.

10. Becerra SP, Sagasti A, Spinella P, et al: Pigment epitheliumderived factor behaves like a non-inhibitory serpin. Nerothrophic activity does not require the serpin reactive loop. J Biol Chem 270: 25992-25999, 1995.

11. Filleur S, Nelius T, de Riese W and Kennedy RC: Characterization of PEDF: a multi-functional serpin family protein. J Cell Biochem 106: 769-775, 2009.

12. Tombran-Tink $\mathbf{J}$ and Johnson LV: Neuronal differentiation of retinoblastoma cells induced by medium conditioned by human RPE cells. Invest Ophthalmol Vis Sci 30: 1700-1707, 1989. 
13. Bouck N: PEDF: anti-angiogenic guardian of ocular function. Trends Mol Med 8: 330-334, 2002.

14. Dawson DW, Volpert OV, Gillis P, et al: Pigment epitheliumderived factor: a potent inhibitor of angiogenesis. Science 285: 245-248, 1999

15. Houenou LJ, D'Costa AP, Li L, et al: Pigment epithelium-derived factor promotes the survival and differentiation of developing spinal motor neurons. J Comp Neurol 412: 506-514, 1999.

16. Abe R, Shimizu T, Yamagishi S, et al: Overexpression of pigment epithelium-derived factor decreases angiogenesis and inhibits the growth of human malignant melanoma cells in vivo. Am J Pathol 164: 1225-1232, 2004.

17. Garcia M, Fernandez Garcia NI, Rivas V, et al: Inhibition of xenografted human melanoma growth and prevention of metastasis development by dual antiangiogenic/antitumour activities of pigment epithelium-derived factor. Cancer Res 64: 5632-5642, 2004.

18. Yang LP, Cheng P, Peng XC, et al: Anti-tumour effect of adenovirus-mediated gene transfer of pigment epithelium-derived factor on mouse B16-F10 melanoma. J Exp Clin Cancer Res 28: 75, 2009.

19. Doll JA, Stellmach VM, Bouck NP, et al: Pigment epitheliumderived factor regulates the vasculature and mass of the prostate and pancreas. Nat Med 9: 774-780, 2003.

20. Filleur S, Volz K, Nelius T, et al: Two functional epitopes of pigment epithelial-derived factor block angiogenesis and induce differentiation in prostate cancer. Cancer Res 65: 5144-5152, 2005.

21. Guan M, Jiang H, Xu C, Xu R, Chen Z and Lu Y: Adenovirusmediated PEDF expression inhibits prostate cancer cell growth and results in augmented expression of PAI-2. Cancer Biol Ther 6: 419-425, 2007.

22. Kojiro M, Hiroki I, Daisuke N, Keisuke H, Kazuhiko N and Katsumi E: Antiangiogenic property of pigment epitheliumderived factor in hepatocellular carcinoma. Hepatology 40 252-259, 2004

23. Uehara H, Miyamoto M, Kato K, et al: Expression of pigment epithelium-derived factor decreases liver metastasis and correlates with favorable prognosis for patients with ductal pancreatic adenocarcinoma. Cancer Res 64: 3533-3537, 2004.

24. Zhang L, Chen J, Ke Y, Mansel RE and Jiang WG: Downregulation of PEDF expression by ribozyme transgene in endothelial and lung cancer cells and its impact on angiogenesis in vitro. Oncol Rep 14: 1615-1619, 2005.

25. Ek ET, Dass CR, Contreras KG and Choong PF: PEDF-derived synthetic peptides exhibit antitumour activity in an orthotopic model of human osteosarcoma. J Orthop Res 25: 1671-1680, 2007.

26. Takenaka K, Yamagishi S, Jinnouchi Y, Nakamura K, Matsui T and Imaizumi T: Pigment epithelium-derived factor (PEDF)induced apoptosis and inhibition of vascular endothelial growth factor (VEGF) expression in MG63 human osteosarcoma cells Life Sci 77: 3231-3241, 2005.
27. Zhang T, Guan M, Xu C, Chen Y and Lu Y: Pigment epitheliumderived factor inhibits glioma cell growth in vitro and in vivo. Life Sci 81: 1256-1263, 2007

28. Volpert OV, Zaichuk T, Zhou W, et al: Inducer-stimulated Fas targets activated endothelium for destruction by anti-angiogenic thrombospondin-1 and pigment epithelium-derived factor. Nat Med 8: 349-357, 2002

29. Yamagishi S, Amano S, Inagaki Y, et al: Pigment epitheliumderived factor inhibits leptin-induced angiogenesis by suppressing vascular endothelial growth factor gene expression through anti-oxidative properties. Microvasc Res 65: 186-190, 2003.

30. Yamagishi S, Inagaki Y, Amano S, et al: Pigment epitheliumderived factor protects cultured retinal pericytes from advanced glycation end product-induced injury through its antioxidative properties. Biochem Biophys Res Commun 296: 877-882, 2002.

31. Cai J, Jiang WG, Grant MB and Boulton M: Pigment epitheliumderived factor inhibits angiogenesis via regulated intracellular proteolysis of vascular endothelial growth fact receptor 1. J Biol Chem 281: 3604-3613, 2006

32. Nathwani AC, Davidoff A, Hanawa H, et al: Factors influencing in vivo transduction by recombinant adeno-associated viral vectors expressing the human factor IX cDNA. Blood 97: $1258-1265,2001$

33. Zhang Z, Zeng G, Zhang L, Xu C and Guo Y: Extracellular domain of kinase domain region mediated by adeno-associated virus inhibits growth and angiogenesis of bladder cancer in Balb-c mice. Chin Med J (Engl) 115: 1209-1212, 2002.

34. Carter PJ and Samulski RJ: Adeno-associated viral vectors as gene delivery vehicles. Int J Mol Med 6: 17-27, 2000.

35. Wu X, Dong X, Wu Z, Cao H, Niu D, et al: A novel method for purification of recombinant adenoassociated virus vectors on a large scale. Chinese Sci Bull 46: 485-488, 2001.

36. Weidner N, Semple JP, Welch WR and Folkman J: Tumour angiogenesis and metastasis - correlation in invasive breast carcinoma N Engl J Med 324: 1-8, 1991.

37. Wang X, Su C, Cao H, et al: A novel triple-regulated oncolytic adenovirus carrying p53 gene exerts poten antitumour efficacy on common human solid cancers. Mol Cancer Ther 7: 1598-1603, 2008.

38. Hase R, Miyamoto M, Uehara H, et al: Pigment epitheliumderived factor gene therapy inhibits human pancreatic cancer in mice. Clin Cancer Res 11: 8737-8744, 2005.

39. Mahtabifard A, Merritt RE, Yamada RE, Crystal RG and Korst RJ: In vivo gene transfer of pigment epithelium-derived factor inhibits tumour growth in syngeneic murine models of thoracic malignancies. J Thorac Cardiovasc Surg 126: 28-38, 2003. 